\title{
Source monitoring and memory confidence in schizophrenia
}

\author{
S. MORITZ, ${ }^{1}$ T. S. WOODWARD AND C. C. RUFF \\ From the Universitätsklinikum Hamburg-Eppendorf, Klinik für Psychiatrie und Psychotherapie, Hamburg, \\ Germany; and Department of Psychology, University of British Columbia, Vancouver and Department of \\ Medicine and Research, Riverview Hospital, Port Coquitlam, British Columbia, Canada
}

\begin{abstract}
Background. The present study attempted to extend previous research on source monitoring deficits in schizophrenia. We hypothesized that patients would show a bias to attribute self-generated words to an external source. Furthermore, it was expected that schizophrenic patients would be overconfident regarding false memory attributions.
\end{abstract}

Method. Thirty schizophrenic and 21 healthy participants were instructed to provide a semantic association for 20 words. Subsequently, a list was read containing experimenter- and self-generated words as well as new words. The subject was required to identify each item as old/new, name the source, and state the degree of confidence for the source attribution.

Results. Schizophrenic patients displayed a significantly increased number of source attribution errors and were significantly more confident than controls that a false source attribution response was true. The latter bias was ameliorated by higher doses of neuroleptics.

Conclusions. It is inferred that a core cognitive deficit underlying schizophrenia is a failure to distinguish false from true mnestic contents.

\section{INTRODUCTION}

In recent years, research on source monitoring in schizophrenia has attracted growing attention (Vinogradov et al. 1997; Keefe et al. 1999). The source monitoring construct has been repeatedly utilized as an interesting theoretical framework for explaining several core features of schizophrenia like hallucinations (Bentall et al. 1991), feelings of alien control (Frith \& Done, 1996) and formal thought disorder (Harvey, 1985; Harvey \& Serper, 1990).

Source monitoring is a class that subsumes different functions rather than an elementary cognitive process. According to Johnson and coworkers (1993) source monitoring involves at least three different abilities: namely, the ability to distinguish one's actual verbal or motor acts

\footnotetext{
1 Address for correspondence: Dr Steffen Moritz, Universitätsklinikum Hamburg-Eppendorf, Klinik für Psychiatrie und Psychotherapie, Martinistrasse 52, D-20246 Hamburg, Germany.
}

from those imagined (internal source monitoring), to distinguish between information from outer sources (external source monitoring) and to distinguish between self-generated and outer verbal or motor actions (reality monitoring).

Deficits in source monitoring are not confined to schizophrenic patients. Failure to identify the source of an event has also been observed in other psychiatric and neurological populations; for example amnestics, subjects who have undergone cingulectomy, obsessive-compulsive (OCD) patients (Ecker \& Engelkamp, 1995) and alcoholics may also exhibit aberrations in source attribution (Johnson et al. 1993). Moreover, source monitoring errors commonly appear in healthy subjects, particularly in elderly people (e.g. Cohen \& Faulkner, 1989) and children (e.g. Foley et al. 1983). Therefore, to demonstrate that patients with schizophrenia have overall difficulties in source monitoring sheds little light on the specific genesis of schizophrenic psychosis. 
Hence, more recent research has attempted to reveal specific response biases and types of source monitoring errors in schizophrenic patients.

Harvey (1985), for example, has inferred an internal source monitoring failure to explain the emergence of formal thought disorder, whereas Bentall et al. (1991) hypothesize that hallucinations arise from a tendency to attribute thoughts/words to an external source (reality monitoring).

The most important aspects of the present experimental approach and the hypotheses they serve are outlined as follows.

(1) In most previous research participants were either instructed to perform a pre-defined task (e.g. Harvey, 1985; Danion et al. 1999; however see also Bentall et al. 1991) or to say/ think a word that was convergently suggested (e.g. Vinogradov et al. 1999). However, it is questionable whether these tasks allow disentanglement of self- from externally-generated items since subjects obeyed externally set rules (i.e. it is rather more appropriate to call these tasks self-executed). For the present study a task was constructed in which verbal productions were truly self-generated. Twenty (affectively neutral) words from the German translation of the KentRosanoff association test (Russell, 1970) were presented to the subject. The participant was required to provide a semantic associate for each stimulus. Despite this different methodological approach, it was expected that the present study would replicate the finding that patients with schizophrenia show significantly greater source attribution and recognition errors than controls (hypothesis 1).

(2) As in other studies, a systematic source attribution bias in schizophrenia was investigated. From the literature it was inferred that schizophrenic patients, especially those with hallucinations, tend to attribute their verbalizations (Bentall \& Slade, 1985; Bentall et al. 1991) to the experimenter (hypothesis 2). It should be noted, however, that previous research has not consistently confirmed an association between source monitoring dysfunctions and hallucinations or positive symptoms (Brébion et al. 1999; Moritz et al. 2001a). At times, negative symptoms (Stirling et al. 1997), thought disorder (Harvey, 1985; Harvey \& Serper, 1990) and hostility (Vinogradov et al. 1997) were also related to source attribution errors. These discrepancies are at least partly explainable by the different experimental approaches adopted.

(3) A major feature of schizophrenic phenomenology is that patients with schizophrenia are convinced that misperceptions/false beliefs are true. For the present study, it was tested whether schizophrenic patients are in general overconfident regarding false source attributions (hypothesis 3). Patients in the present study were asked to assess how confident they were with respect to source judgements (for both wrong and right responses) prior to feed-back by the experimenter. No predictions were made as to whether this deficit reflects a state-like dysfunction or occurs in all schizophrenic patients.

Moreover, the contribution of other cognitive disturbances on source monitoring errors was assessed. Johnson et al. (1993) emphasize the importance of memory and executive dysfunctions on source attribution errors. For example, external input is often more vivid and perceptual than imaginations. Many healthy persons make their internal source monitoring distinctions on the basis of the richness of their memories, that is more detailed and perceptual mnestic contents are taken as suggestive that an event was previously perceived and not imagined. However, memory dysfunctions seen in schizophrenic patients (see Aleman et al. 1999, for a recent meta-analysis) may lead to an impoverishment of stored external events and thus may decrease qualitative differences between internally generated and perceived items which in turn may give rise to misattributions.

In addition, executive dysfunctions may also play a role for memory attribution where source attributions cannot definitely be made according to heuristic analyses (see above). It is important to note that these deliberate processes may not only aid automatic attributions but at times may also challenge fast-acting (heuristic) attributions and vice versa (Johnson et al. 1993). Since both memory and executive functions may be linked to source memory deficits, and since schizophrenic patients show strong differential deficits in both executive and memory tasks (e.g. Moritz et al. 2002), we wanted to explore whether source memory errors are an epiphenomenon of disturbances in these more basic neuropsychological functions.

It should be noted that at least the research hypotheses 2 (bias towards externalizing 
Table 1. Sociodemographic and psychopathological characteristics of the samples

\begin{tabular}{|c|c|c|c|}
\hline & $\begin{array}{l}\text { Healthy subjects } \\
\qquad(N=21)\end{array}$ & $\begin{array}{l}\text { Schizophrenic subjects } \\
\qquad(N=30)\end{array}$ & Statistics \\
\hline Age & $27 \cdot 00(10 \cdot 7)$ & $31 \cdot 08(8 \cdot 3)$ & $t(43)=1 \cdot 62 ; \mathrm{NS}$ \\
\hline Sex (male/female) & $11 / 10$ & $21 / 9$ & $\chi^{2}(1)=1 \cdot 64 ; \mathrm{NS}$ \\
\hline Years of school education & $11.53(1.7)$ & $11.96(1.8)$ & $t(43)=0.83 ; \mathrm{NS}$ \\
\hline Pre-morbid intelligence (IQ) & $112 \cdot 93(14 \cdot 0)$ & $112 \cdot 67(12 \cdot 8)$ & $t(37)=0 \cdot 06 ; \mathrm{NS}$ \\
\hline Previous hospitalizations & - & $2 \cdot 65(4 \cdot 0)$ & - \\
\hline Length of illness & - & $4 \cdot 48(6 \cdot 0)$ & - \\
\hline Chlorpromazine equivalent dosage in $\mathrm{mg}$ & - & $253 \cdot 04(189 \cdot 7)$ & - \\
\hline \multicolumn{4}{|l|}{ BPRS } \\
\hline Positive syndrome & - & $8 \cdot 86(4 \cdot 4)$ & - \\
\hline Negative syndrome & - & $7 \cdot 62(3 \cdot 0)$ & - \\
\hline Disorganization & - & $3 \cdot 28(1 \cdot 65)$ & - \\
\hline Total (18 items) & - & $37 \cdot 03(12 \cdot 3)$ & - \\
\hline
\end{tabular}

self-generated items towards the experimenter) and 3 (hyper-confidence for false attributions) cannot easily be accounted for by a generalized performance deficit. General cognitive dysfunction implies quantitatively diminished objective scores in a forced-choice paradigm such as ours but does not explain a systematic source attribution bias or hyper-confidence regarding misattributions (many patients with mnestic dysfunctions including demented patients rather mistrust even true memories; see also Zitterl et al. 1999, for OCD patients).

\section{METHOD}

\section{Subjects}

Thirty schizophrenic in-patients from the university hospital of Hamburg (Germany) participated in the study. Patients had been in hospital for 2 weeks following acute psychotic exacerbation. All patients were on atypical neuroleptic mono-therapy for 2 weeks following a wash-out period of at least 3 days. Diagnoses on in-patients were made by experienced physicians and by a medical student prior to neurocognitive assessment. Diagnoses relied on DSM-IV criteria. Furthermore, careful screening of medical records ensured exclusion of patients who had a history of severe neurological disorder, substance abuse and another major axis 1 disorder. No published semi-structured diagnostic interview was performed. Current psychopathological symptoms were assessed through the Brief Psychiatric Rating Scale (BPRS, 18 items) (Overall \& Gorham, 1988). Psychopathological assessment was made blind to neurocognitive status (i.e. the neuropsychological experimenter never performed the psychopathological ratings).
The negative syndrome was determined by the sum of the item scores for 'emotional withdrawal', 'motor retardation' and 'blunted affect'. The positive syndrome was composed from the following items: 'unusual thought content', 'grandiosity', 'suspiciousness' and 'hallucinatory behaviour'. The disorganized syndrome comprised only two symptoms: 'conceptual disorganization' and 'mannerisms and posturing'. Syndrome composition followed previous factor analytical solutions (e.g. Frith \& Johnstone, 1996; Moritz et al. 2001 b). Pre-morbid intelligence was estimated by a multiple-choice vocabulary test (Lehrl, 1995).

Controls were drawn from hospital staff and the general population. Initially, 22 healthy subjects screened for psychopathological disturbances participated. One subject was later excluded due to a history of severe substance abuse. Sociodemographic and psychopathological characteristics of the sample are displayed in Table 1. As can be seen, patients had only minor to moderate symptoms. All subjects gave written informed consent to participate after they had been fully informed about the study.

\section{Procedure}

\section{Learning phase}

Twenty words from the Kent-Rosanoff association test were read to the subject by the experimenter. After each word, the subject was instructed to give his/her first association (one single word, no phrases, no names, no repetition of previous words). Subjects were told that later they would be asked to recall these words (both given and self-generated words). 


\section{Recognition phase}

Ten minutes after the learning phase, 60 words were read to the subject. Words belonged to the following four conditions (words were presented in random order): (1) 20 words from the KentRosanoff association test; (2) 20 words produced by the participant in response to 1 ; (3) 10 new words with no associative relation with 1 or 2 ; and, (4) 10 new words that were related but never identical with 1 or 2 (e.g. if the prime word was 'bread' and the subject associated 'sausage', the related new word could be 'cheese'). There were always two words for each stimulus in case the subject's association (i.e. condition 2) matched the first related word. The related new words were highly frequent associations to the prime words according to the German translation and norm study of the Kent-Rosanoff association test (Russell, 1970).

After each presented word the subject was instructed to provide three responses: (a) recognition, new or old word; $(b)$ source attribution, if the word was recognized as being old, it had to be determined who said it: the experimenter or the subject; $(c)$ memory confidence, subjects were instructed to rate on a four point scale how certain they were concerning their source attribution judgement ( $1=$ don't know; $2=$ rather uncertain; $3=$ rather certain; $4=$ convinced). After these responses were recorded, subjects were given feedback whether their answer was right or not (e.g. if 'foot' (given by the experimenter) was read to the subject and the subject answered 'old, I said it and I am convinced' the experimenter would say 'No, I said that word').

\section{Further neuropsychological tasks}

To assess the impact of verbal memory and executive functioning on source monitoring, patients were also administered the Rey-Auditory Verbal Learning Test (RAVLT, verbal memory, Lezak, 1995) and a computerized version of the Wisconsin Card Sorting Test (WCST, executive functioning, Heaton, 1981). For the present analyses, the WCST parameters 'categories completed' (0-6) and 'number of perseverative errors' were extracted as well as the RAVLT long-term recall parameter (number of items recalled twenty minutes after the last presentation of a list of 15 items).

\section{RESULTS}

For all analyses described in the following sections, the significance level was set at $5 \%$, twotailed. All indices were correlated with the three psychopathological syndrome scores (see above) as well as the single scores for hallucinations, delusions and formal thought disorder. Only significant correlations or results from a priori hypotheses are reported for psychopathology.

\section{Sociodemographic variables}

Patients and controls did not differ regarding sex distribution, age, pre-morbid intelligence and years of school (see Table 1).

\section{Associative strength}

All words generated by the subjects were scored according to their relative frequency, which was derived from German norm scores (Russell, 1970) (e.g. if a subject said 'hand' to the prime 'foot' the value 38 was assigned since 38 persons out of the 300 subject sample of Russell had the same association). Moreover, the ranks for the associations were computed (e.g. if a subject associated 'fast' to the prime 'slow', he/she achieved score 1 since most subjects in the norm study made the same association). Patients and controls were comparable regarding the strength of associations for both relative rank (healthy subjects, mean $=9 \cdot 21$ (s.D. $=4 \cdot 0)$; schizophrenic subjects, mean $=10 \cdot 07$ (s.D. $=4 \cdot 2), t(49)=0 \cdot 74$, $P>0.4$ ) and normed word frequency healthy subjects, mean $=52.72$ (s.D. $=14 \cdot 8)$; schizophrenic subjects, mean $=52 \cdot 61$ (s.D. $=13 \cdot 6), t(49)=0 \cdot 03$, $P>0.9)$.

\section{Source monitoring task}

Prior to conducting $t$ test comparisons between schizophrenic and healthy subjects a two-way ANOVA was computed using group (healthy controls, schizophrenic subjects) as the betweensubject variable and condition (self-generated, experimenter-generated, unrelated new, related new) as the within-subject variable. Percentage of correctly identified items served as dependent variable. The effects for group $(F(1,49)=8 \cdot 05$, $P \leqslant 0.005)$ and condition $(F(3,147)=52.67$, $P \leqslant 0.001)$ achieved significance. The significant group effect reflects lower percentage of correct responses in subjects with schizophrenia. The highly significant condition effect was further 
Table 2. Means and standard deviations of source monitoring and recognition errors ( $\mathrm{t}$ test differences are displayed in the cells of the schizophrenic participants)

\begin{tabular}{|c|c|c|c|c|c|}
\hline \multirow[b]{4}{*}{ Group and attributed origin } & \multicolumn{4}{|c|}{ Number of responses: actual source } & \multirow[b]{4}{*}{ Row total (errors) } \\
\hline & \multicolumn{2}{|c|}{ Old words } & \multicolumn{2}{|c|}{ New words } & \\
\hline & Experimenter & Self & Unrelated & Related & \\
\hline & Mean (s.D.) & Mean (s.D.) & Mean (S.D.) & Mean (s.D.) & \\
\hline \multicolumn{6}{|l|}{ Normal subjects } \\
\hline Experimenter & $17 \cdot 52(1 \cdot 6)$ & $2 \cdot 38(1 \cdot 5)$ & $0.05(0.2)$ & $0.24(0 \cdot 4)$ & $2 \cdot 67(1.7)$ \\
\hline Self & $1 \cdot 19(1 \cdot 4)$ & $15 \cdot 52(1.9)$ & $0 \cdot 10(0 \cdot 3)$ & $0 \cdot 14(0 \cdot 4)$ & $1 \cdot 43(1 \cdot 7)$ \\
\hline New & $1 \cdot 29(1 \cdot 2)$ & $2 \cdot 10(1 \cdot 3)$ & $9 \cdot 85(0 \cdot 4)$ & $9 \cdot 62(0 \cdot 5)$ & $3 \cdot 39(2 \cdot 0)$ \\
\hline Total recognition errors & $1 \cdot 29(1 \cdot 2)$ & $2 \cdot 10(1 \cdot 3)$ & $0 \cdot 15(0 \cdot 4)$ & $0 \cdot 38(0 \cdot 5)$ & $3.92(1.9)$ \\
\hline Column total (errors) & $2 \cdot 48(1.6)$ & $4 \cdot 48(1 \cdot 8)$ & $0 \cdot 15(0 \cdot 4)$ & $0 \cdot 38(0 \cdot 5)$ & $7 \cdot 49(3 \cdot 0)$ \\
\hline \multicolumn{6}{|l|}{ Schizophrenic subjects } \\
\hline Experimenter & $16 \cdot 27(3 \cdot 0) \dagger$ & $3 \cdot 23(2 \cdot 1)$ & $0 \cdot 20(0 \cdot 6)$ & $0.97(1 \cdot 1)^{* *}$ & $4.40(2 \cdot 8)^{* *}$ \\
\hline Self & $1 \cdot 83(1 \cdot 5)$ & $14 \cdot 10(3 \cdot 5) \dagger$ & $0 \cdot 03(0 \cdot 2)$ & $0 \cdot 13(0 \cdot 4)$ & $2 \cdot 00(1 \cdot 5)$ \\
\hline New & $1.90(2 \cdot 5)$ & $2 \cdot 67(2 \cdot 6)$ & $9 \cdot 77(0 \cdot 6)$ & $8.90(1 \cdot 2)^{* *}$ & $4 \cdot 57(4 \cdot 8)$ \\
\hline Total recognition errors & $1.90(2 \cdot 5)$ & $2 \cdot 67(2 \cdot 6)$ & $0 \cdot 23(0 \cdot 6)$ & $1 \cdot 10(1 \cdot 2)^{* *}$ & $5 \cdot 90(4 \cdot 6)^{*}$ \\
\hline Column total (errors) & $3.73(3.0) \dagger$ & $5.90(3 \cdot 5) \dagger$ & $0 \cdot 23(0 \cdot 6)$ & $1 \cdot 10(1 \cdot 2)^{* *}$ & $10 \cdot 97(6 \cdot 0)^{* *}$ \\
\hline
\end{tabular}

$\dagger P \leqslant 0 \cdot 1 ; * P \leqslant 0 \cdot 05 ; * * \quad P \leqslant 0 \cdot 01$, two-tailed.

Values in italics indicate correct responses; all other values represent errors.

differentiated through planned contrasts which showed that all conditions differed regarding percentage of right responses (at least $P \leqslant 0.005$; subjects made the least errors in the condition with new, unrelated words). Contrary to expectation (see discussion), subjects recognized experimenter-generated words significantly better than self-generated words $(P \leqslant 0 \cdot 001)$. The group $\times$ condition interaction was not significant.

\section{Recognition and source attribution errors}

As can be derived from Table 2, recognition errors for the old conditions (i.e. words generated by the experimenter or subject) were those incorrectly labelled by the subject as new. In contrast, for the two new conditions, recognition errors were the sum of the errors for attributing new words to either the experimenter or to oneself. Schizophrenic subjects $($ mean $=5 \cdot 90)$ showed overall more recognition errors than controls $($ mean $=3.92) \quad(t(49)=2 \cdot 13, P \leqslant 0 \cdot 05)$. Specifically, schizophrenic subjects more often regarded new words that were related to old words as already heard (healthy subjects, mean $=0.38$; schizophrenic subjects, mean $=1 \cdot 10, t(49)=$ $2 \cdot 97, P \leqslant 0 \cdot 005)$. This tendency was significantly correlated with severity of disorganization symptoms $(r(28)=0.40, \quad P=0.03)$, most notably formal thought disorder $(r(28)=0.50$, $P=0 \cdot 006)$.
Overall errors (recognition and source attribution errors) were increased in schizophrenic patients $(t(49)=2.74, P \leqslant 0.01$; schizophrenic subjects, mean $=10.97$ (s.D. $=6.0$ ) ; healthy subjects, mean $=7.49$ (S.D. $=3.0)$ ). To disentangle recognition and source monitoring errors, we computed a sum score for those conditions in which recognition but not source attribution was correct (i.e. self-generated items that were misattributed to the experimenter and externally generated items that were misattributed to oneself; please note, for both error types words were correctly identified as old). Again, schizophrenic subjects performed worse than controls $(t(49)=1.98, P \leqslant 0.05)$. Hypothesis 1 therefore was confirmed. Hallucinations were not correlated with the tendency to attribute own verbalizations to the experimenter $(r=0 \cdot 16, \mathrm{NS})$. Analyses of variance with subsequent simple contrasts also revealed no significant differences between hallucinators (BPRS item 'hallucinatory behaviour', score $\geqslant 3, N=12$ ), non-hallucinators and controls $(F(2,49)=1 \cdot 87, P>0 \cdot 1)$ regarding this response tendency (hallucinators, mean $=$ 3.66 (s.D. $=1 \cdot 44$ ); non-hallucinators, mean $=3.06$ $($ S.D. $=2 \cdot 46)$; controls, mean $=2 \cdot 38($ s.D. $=1 \cdot 50)$ ). Hypothesis 2 therefore was dismissed.

A significant bias emerged for schizophrenic patients to attribute words to the experimenter $(t(49)=2 \cdot 74, P \leqslant 0 \cdot 01)$. This bias was negatively 
Table 3. Means and standard deviations for subjective source attribution confidence

\begin{tabular}{|c|c|c|c|}
\hline & $\begin{array}{l}\text { Healthy subjects }(N=21) \\
\text { Mean (s.D.) }\end{array}$ & $\begin{array}{l}\text { Schizophrenic subjects }(N=30) \\
\text { Mean (S.D.) }\end{array}$ & $t_{(49)} P$ \\
\hline Subjective confidence for correct responses & $3 \cdot 75(0 \cdot 2)$ & $3 \cdot 71(0 \cdot 4)$ & $0 \cdot 41 \mathrm{NS}$ \\
\hline Subjective confidence for incorrect responses & $2 \cdot 62(0 \cdot 5)$ & $3 \cdot 16(0 \cdot 7)$ & $2 \cdot 93 * *$ \\
\hline Difference in source attribution confidence & $1 \cdot 13(0 \cdot 4)$ & $0.55(0 \cdot 6)$ & $3 \cdot 78^{* * *}$ \\
\hline
\end{tabular}

** $P<0 \cdot 01 ;$ *** $P<0 \cdot 001$; NS, not significant.

associated with negative symptoms $(r(28)=$ $-0 \cdot 42, P=0 \cdot 02)$. Closer inspection revealed that this tendency was significant only for new, related words but not for self-generated nor unrelated, new words (see Table 2 : healthy subjects, mean $=0 \cdot 24$; schizophrenic subjects, mean $=$ $0 \cdot 97)$. Recognition errors were not significantly correlated with core source monitoring errors (i.e. sum of self-generated words misattributed to the experimenter and experimenter-generated words to oneself; $r(49)=0 \cdot 23, \mathrm{NS})$.

\section{Source confidence}

Each subject's confidence in their source attribution rating was summed up separately for correct responses and incorrect responses, respectively. Healthy controls were slightly more confident for responses that later turned out to be correct (see Table 3). However this difference did not achieve significance. For responses that were incorrect, patients $($ mean $=3 \cdot 16)$ showed significantly greater confidence than controls (mean $=2.62, t(49)=2.93, \quad P \leqslant 0.005)$; ratings given by patients corresponded approximately to the value 'rather certain'.

It was also tested whether the difference between confidence ratings for right and wrong answers achieved significance. This served as an index for discriminability between true and false source memories. Both groups were significantly more confident for right than for wrong memory contents (for both groups at least $P \leqslant$ 0.001). However, as expected from hypothesis 3, patients, in comparison with controls, had significantly lower difference scores (i.e. controls separated true from false source memories with greater confidence). No confidence parameter correlated with any of the syndrome scores.

\section{Medication effects}

Neuroleptic medication was converted in chlorpromazine equivalent doses according to algorithms proposed by Dietmaier \& Laux (1998).
When the schizophrenic sample was median split according to chlorpromazine equivalent dosage (i.e. $225 \mathrm{mg}$ ), patients receiving higher doses showed a significantly greater difference between confidence for right responses relative to confidence for wrong responses $(t(28)=2 \cdot 35, P \leqslant$ $0 \cdot 05)$. No other neurocognitive index was related to medication.

\section{Correlational analyses with WCST, RAVLT and background variables}

The correlational analyses with major sociodemographic and neuropsychological data (WCST, RAVLT) were restricted to those memory parameters which were significant and consistent discriminators of schizophrenic patients and controls.

The two WCST parameters were not correlated with source monitoring or recognition. Years of school, age, gender and premorbid intelligence as assessed with a vocabulary test were not correlated with mal-performance in any of the parameters. Long-term recall as measured with the RAVLT correlated with recognition deficits $(r(49)=-0 \cdot 47, P \leqslant 0 \cdot 01)$.

\section{DISCUSSION}

The present results confirm previous research reporting that, relative to controls, schizophrenic subjects achieve poorer source memory (Vinogradov et al. 1997; Keefe et al. 1999) and recognition scores (Moritz et al. 2001 a). Hypothesis 1 was thus confirmed. However, as already noted, deficits in source monitoring are also common in other populations (Johnson \& Raye, 1981; Johnson et al. 1993), so that compromised source monitoring abilities cannot explain why a subject has schizophrenia and not another psychiatric disorder. Moreover, in a forced-choice design such as ours global memory problems consistently found in schizophrenia (Moritzet al. $2001 a$; see Aleman et al. 1999, for a meta-analysis) 
imply decreased overall source memory scores, since subjects are asked to provide a response for all items (Keefe et al. 1999). To address this issue, we computed an index representing attribution errors that were not accompanied by recognition errors. Although the group difference achieved significance, the strength of the difference was quite small, so that again it is doubted that group differences will occur when comparing schizophrenic subjects to psychiatric or neurological controls.

Unexpected from previous research (Bentall et al. 1991) hallucinating schizophrenic patients did not exhibit a significantly increased tendency to attribute self-generated verbal productions to the experimenter (hypothesis 2 was thus not confirmed). For the entire schizophrenic sample, compared to the control group, this tendency was increased, but the group difference failed to reach significance. This result has recently been confirmed by our work group (Moritz \& Woodward, 2002). While our results speak against a global externalization bias in hallucinating patients (i.e. which can be demonstrated with neutral material), there is evidence that hallucinatory patients show an externalization bias for emotionally valenced material (Morrison \& Haddock, 1997). In a current project, we therefore, employ positively and negatively valenced words along with neutral words.

We consider the most essential finding of the present study to be that patients were more convinced than controls with regard to the correctness of their false attributions (hypothesis 3 was confirmed). Nevertheless, patients, like controls, were significantly more confident about the correctness of their true memories than their wrong attributions. The latter result is an indication that patients did understand the task. Also, patients were not generally biased towards being more confident (ratings for correct attributions did not differ significantly from controls). It is also important to note that previous research has shown that schizophrenic patients are, for the most part, able to assess their cognitive problems adequately (Cuesta et al. 1996; Moritz et al. 2001 c) so that it is assumed that confidence ratings were valid.

As a working hypothesis, we propose that schizophrenic patients in general might not adequately distinguish true from false source memory episodes. It is interesting that this deficit was not correlated with positive (reality distortion) symptoms. This may hint at the possibility that all patients who currently reveal, or once have displayed psychotic symptoms, share a latent deficit. As already stressed, controls also committed source monitoring errors. However, it seems that healthy subjects attach some kind of 'not trustworthy' tag to these memories, which separates them from fact knowledge. Schizophrenic patients, on the other hand, seem less able to tease apart fictive events from true memories, so that fact and fiction cannot be entirely disentangled.

An important but unexpected finding that requires further inspection was that neuroleptics seem to increase the confidence gap between true and false attributions. When being replicated, this finding may give insight into how antipsychotic drugs functionally ameliorate neurocognitive deficits underlying psychosis. However, the present results were statistically weak, and the dosage range rather narrow, so caution in interpretation is clearly warranted. Furthermore, medication effects on neurocognition are best explored using an experimental design in which dosage is manipulated independent from symptomatology.

The present findings share some resemblance with results obtained by Danion et al. (1999). Danion et al. required patients to provide 'know' or 'remember' ratings for source memory responses. In the light of our data, it is especially interesting that for their schizophrenic sample the frequency of 'remember' responses was equal for correct and for incorrect responses (i.e. wrong responses were equally 'perceptual' as false answers). However, the know-remember assessment cannot be equated with the present confidence assessment: Danion et al. incorporated no 'guess' rating so that the sub-optimal 'know' response may partly reflect guessing (see also Tien, 1999) as well as high-confident responses which, however, lack a conscious mnestic image of the learning episode.

Schizophrenic patients, particularly those with elevated disorganized symptoms, were strongly biased to believe that new, but related to old information (i.e. words that were associated with both prime words and self-generated associates) was initially presented (recognition error). Specifically, patients believed the experimenter produced that information. This pattern of 
results can be considered an extreme variant of the 'it-had-to-be-you-effect' (Johnson \& Raye, 1981) also seen in many normals, whereby new information that is only weakly memorized is rather attributed to an external source than to oneself. A promising line of research has suggested that the primary cognitive dysfunction underlying the disorganization syndrome is fastand far-spreading neural activation in semantic memory (Spitzer et al. 1993; Spitzer, 1997; Moritz et al. 2001d). This hyper-activation of semantics results in an increased ease in the production of word associations for disorganized patients. Greater-than-normal activation of related information may thus lead to the misperception that these words have been actually heard.

Another somewhat unexpected finding was that neither controls nor schizophrenic participants showed a generation effect (subjects normally recall self-generated words better than experimenter-generated words: see Slamecka \& Graf, 1978; Johnson \& Raye, 1981). On the contrary, subjects recognized significantly more information provided by the experimenter than by themselves (see Harvey, 1985, for a similar finding). As this pattern of results was evident for both groups and has been replicated by subsequent research in our laboratory, we do not think that this is a chance finding and suggest further exploration. Possible moderators of this effect might be that self- and externallygenerated information was closely related, leading to high source ambiguity. Secondly, the words from the recall list were read by the experimenter. If the reversed generation effect is attributable to an advantage for words that are re-read by the same source, the effect should be abolished when a third person speaks all words in the recognition list. However, if the effect reflects a disadvantage for self-generated words that are verbally usurpated by an external source the effect might remain and the usual pattern will only appear when the information is presented neutrally (e.g. with written recognition lists).

The present result profile needs further replication and elaboration. While the present study may have elucidated some candidate mechanism for the emergence of psychosis, other factors (e.g. stress resistance, affect; see Morrison \& Haddock, 1997) remain to be incorporated into this model and may enhance its validity.
Moreover, it will be of major importance to reveal whether these abnormalities are specific to schizophrenia.

Currently, we are attempting to replicate the present findings; we will more thoroughly collect psychopathological data using an instrument called PANADSS (Andresen \& Moritz, 2000; Moritz et al. 2001 c), which allows the separation of different forms of hallucinations and delusions. It will also be assessed whether patients had ever experienced symptoms of reality distortion such as hallucinations and delusions. It may be the case that those patients who have never experienced overt positive symptoms do not exhibit hyper-confidence for misattributions.

\section{REFERENCES}

Aleman, A., Hijman, R., de Haan, E. H. F. \& Kahn, R. S. (1999) Memory impairment in schizophrenia: a meta-analysis. American Journal of Psychiatry 156, 1358-1366.

Andresen, B. \& Moritz, S. (2000). Positive and Negative and Disorganized Symptoms Scale for Schizophrenia (PANADSS). Manual. PPV: Westerau.

Bentall, R. P. \& Slade, P. D. (1985). Reality testing and auditory hallucinations: a signal detection analysis. British Journal of Clinical Psychology 24, 159-169.

Bentall, R. P., Baker, G. A. \& Havers, S. (1991). Reality monitoring and psychotic hallucinations. British Journal of Clinical Psychology 30, 213-222.

Brébion, G., Amador, X., Smith, M. J., Malaspina, D., Sharif, Z. \& Gorman, J. M. (1999). Opposite links of positive and negative symptomatology with memory errors in schizophrenia. Psychiatry Research 88, 15-24.

Cohen, G. \& Faulkner, D. (1989). Age differences in source forgetting: effects on reality monitoring and on eye witness testimony. Psychology and Aging 4, 10-17.

Cuesta, M. J., Peralta, V. \& Juan, J. A. (1996). Abnormal subjective experiences in schizophrenia: its relationships with neuropsychological disturbances and frontal signs. European Archives of Psychiatry and Clinical Neuroscience 246, 101-105.

Danion, J.-M., Rizzo, L. \& Bruant, A. (1999). Functional mechanisms underlying impaired recognition memory and conscious awareness in patients with schizophrenia. Archives of General Psychiatry 56, 639-644

Dietmaier, O. \& Laux, G. (1998). Übersichtstabellen. In NeuroPsychopharmaka, Band 4 (ed. P. Riederer, G. Laux and W. Pöldinger), pp. 505-522. Springer: Wien.

Ecker, W. \& Engelkamp, J. (1995). Memory for actions in obsessivecompulsive disorder. Cognitive Psychotherapy 23, 349-371.

Foley, M. A., Johnson, M. K. \& Raye, R. C. (1983). Age-related changes in confusion between memories for thoughts and memories for speech. Child Development 54, 51-60.

Frith, C. D. \& Done, D. J. (1989). Experiences of alien control in schizophrenia reflect a disorder in the central monitoring of action. Psychological Medicine 19, 359-363.

Frith, C. D. \& Johnstone, E. C. (1996). Validation of three dimensions of schizophrenic symptoms in a large unselected sample of patients. Psychological Medicine 26, 669-689.

Harvey, P. D. (1985). Reality monitoring in mania and schizophrenia. The association of thought disorder and performance. Journal of Nervous and Mental Disease 173, 67-73.

Harvey, P. D. \& Serper, M. R. (1990). Linguistic and cognitive failures in schizophrenia. A multivariate analysis. Journal of Nervous and Mental Disease 178, 487-494. 
Heaton, R. K. (1981). Wisconsin Card Sorting Test Manual. Psychological Assessment Resources Inc.: Odessa, FL.

Johnson, M. K. \& Raye, C. L. (1981). Reality monitoring. Psychological Review 88, 67-85.

Johnson, M. K., Hashtroudi, S. \& Lindsay, D. S. (1993). Source monitoring. Psychological Bulletin 114, 3-28.

Keefe, R. S. E., Arnold, M. C., Bayen, U. J. \& Harvey, P. D. (1999). Source monitoring deficits in patients with schizophrenia; a multinomial modelling analysis. Psychological Medicine 29, 903-914.

Lehrl, S. (1995). Mehrfachwahl-Wortschatz-Intelligenztest: MWT-B. PERIMED-spitta.: Balingen, Germany.

Lezak, M. D. (1995). Neuropsychological Assessment, Vol. 3. edn. Oxford University Press: Oxford.

Moritz, S. \& Woodward, T. S. (2002). Memory confidence and false memories in schizophrenia. Journal of Nervous and Mental Disease 190, 641-643.

Moritz, S., Heeren, D., Andresen, B. \& Krausz, M. (2001a). An analysis of the specificity and the syndromal correlates of verbal memory impairments in schizophrenia. Psychiatry Research 101, $23-31$.

Moritz, S., Andresen, B., Jacobsen, D., Mersmann, K., Wilke, U., Lambert, M., Krausz, M. \& Naber, D. (2001 b). Neuropsychological correlates of schizophrenic syndromes in patients treated with atypical neuroleptics. European Psychiatry 16, 354-361.

Moritz, S., Lambert, M., Andresen, B., Böthern, A., Naber, D. \& Krausz, M. $(2001 c)$. Subjective cognitive dysfunction in firstepisode and chronic schizophrenic patients. Comprehensive Psychiatry 42, 213-216.

Moritz, S., Mersmann, K., Jacobsen, D., Kloss, M., Andresen, B., Pawlik, K. \& Naber, D. (2001d). Enhanced semantic priming in thought-disordered schizophrenic patients using a word pronunciation task. Schizophrenia Research 48, 303-308.

Moritz, S., Mersmann, K., Kloss, M., Jacobsen, D., Wilke, U., Andresen, B., Naber, D. \& Pawlik, K. (2001e). Further evidence for 'hyper-priming' in thought-disordered schizophrenics. Psychological Medicine 31, 221-229.
Moritz, S., Kloss, M., Jahn, H., Hand, I., Haasen, C. \& Krausz, M. (2002). Executive functioning in obsessive-compulsive disorder, unipolar depression and schizophrenia. Archives of Clinical Neuropsychology 17, 447-483.

Morrison, A. P. \& Haddock, G. (1997). Cognitive factors in source monitoring and auditory hallucinations. Psychological Medicine 27, 669-679.

Overall, J. E. \& Gorham, D. R. (1988). The Brief Psychiatric Rating Scale (BPRS): recent developments in ascertainment and scaling. Psychopharmacology Bulletin 24, 97-99.

Russell, W. A. (1970). The complete German language norms for responses to 100 words from the Kent-Rosanoff word association test. In Norms of Word-Association (ed. L. Postman and G. Keppel), pp. 53-94. Academic Press: New York.

Slamecka, N. J. \& Graf, P. (1978). The generation effect: delineation of a phenomenon. Journal of Experimental Psychology: Human Learning and Memory 4, 592-604.

Spitzer, M. (1997). A cognitive neuroscience view of schizophrenic thought disorder. Schizophrenia Bulletin 23, 29-50.

Spitzer, M., Braun, U., Hermle, L. \& Maier, S. (1993). Associative semantic network dysfunction in thought-disordered schizophrenic patients: direct evidence from indirect semantic priming. Biological Psychiatry 34, 864-877.

Stirling, J. D., Hellewell, J. S. E. \& Hewitt, J. (1997). Verbal memory impairment in schizophrenia: no sparing of short-term recall. Schizophrenia Research 25, 85-95.

Tien, A. Y. (1999). Deficits of information management associated with schizophrenia. Awareness and associated integrative cognitive functions. Archives of General Psychiatry 56, 647-648.

Vinogradov, S., Willis-Shore, J., Poole, J. H., Marten, E., Ober, B. A. \& Shenaut, G. K. (1997). Clinical and neurocognitive aspects of source monitoring errors in schizophrenia. American Journal of Psychiatry 154, 1530-1537.

Zitterl, W., Urban, C., Linzmayer, L., Aigner, M., Demal, U. \& Zitterl-Eglseer, K. (1999). Memory functioning in obsessive compulsive disorder. CNS Spectrums 4, 22. 